\title{
Evaluation of Fetal Pulmonary Veins During Early Gestation by Pulsed Doppler Ultrasound: A Feasibility Study
}

\author{
Aldo L. Schenone ${ }^{1}$ (D) Gelsy Giugni ${ }^{2} \cdot$ Mauro H. Schenone $^{3} \cdot$ Luis Diaz $^{2}$ • \\ Alberto Bermudez $^{2} \cdot$ David Majdalany $^{4} \cdot$ Alberto Sosa-Olavarria $^{2}$
}

Received: 25 March 2015 / Accepted: 19 May 2015/Published online: 4 June 2015

(C) Society of Fetal Medicine 2015

\begin{abstract}
Congenital heart disease (CHD) is the leading cause of infant mortality. Nevertheless, routine prenatal ultrasound only detects $30 \%-50 \%$ of CHD cases. Therefore, emphasis should be placed on early predictors of CHD that allow physicians to timely refer high-risk patient to specialized fetal echocardiography, in order to maximize chances of improved outcomes. The fetal venous system has been the focus of attention as a marker of heart function. Yet, there exist inconsistent data about the acquisition of pulmonary venous flow in early pregnancy. This study aims to determine the feasibility of fetal pulmonary venous flow acquisition in early pregnancy, and the association between pulmonary vein (A-wave) flow reversal and the presence of CHD. Total of 211 consecutive pregnant women, between 12 and 15 weeks of gestation, underwent routine obstetric ultrasound along with basic fetal echocardiography including fetal pulmonary venous flow assessment. The pulmonary venous flow velocity waveforms were acquired by either Doppler or Doppler plus enhance flow technique based on equipment availability. All the cases were re-evaluated by specialized fetal echocardiography during late pregnancy to identify congenital heart anomalies. The acquisition of fetal pulmonary
\end{abstract}

Aldo L. Schenone

a.schenone@gmail.com

1 Internal Medicine Department, Cleveland Clinic Foundation, 9500 Euclid Avenue, NA10, Cleveland, OH 44195, USA

2 Centro de Estudios Ultraosnograficos Perinatales, Valencia, Venezuela

3 Department of Materno-fetal Medicine, University of Tennessee, Memphis, TN, USA

4 Heart and Vascular Institute, Cleveland Clinic, Cleveland, $\mathrm{OH}$, USA vein, between 12 and 15 weeks of gestation, was successfully accomplished in $87.7 \%$ of cases. Five out of seven fetuses with end-diastolic (A-wave) pulmonary venous flow reversal were associated with confirmed cardiac anomaly. In this pilot study, pulmonary venous (A-wave) flow reversal was associated with cardiac anomalies in $86.8 \%$ of cases $(\mathrm{p}=0.001)$. This pilot study proposes that Doppler interrogation of fetal pulmonary vein flow during early pregnancy is feasible. Furthermore, the presence of pulmonary vein (A-wave) reversal might represent a marker of major cardiac anomalies. Further investigation is needed to confirm these findings.

Keywords Fetal pulmonary veins - Congenital cardiac anomaly $\cdot$ Congenital heart disease $\cdot$ First trimester screening $\cdot$ Fetal heart disease screening

\section{Introduction}

Congenital heart disease (CHD) is the most common congenital malformation and the leading cause of infant mortality $[1,2]$. Despite evidence that early detection of certain heart malformations could lead to improved fetal/neonatal outcomes [3], routine prenatal ultrasound only detects approximately $30 \%-50 \%$ of CHD cases [4, 5]. While many cardiac abnormalities are missed during routine ultrasound, most of them can be detected by specialized fetal echocardiography in late pregnancy [2]. Yet, only about $26 \%$ of fetuses can be fully evaluated in early gestation [6]. Therefore, emphasis should be placed on early predictors of CHD that allow physicians to timely refer high-risk patients to specialized fetal echocardiography, in order to maximize chances of improved prenatal/neonatal outcomes. 
The fetal venous system has recently been the focus of attention since changes on the venous flow might indirectly reflect variations in heart performance [7]. The normal fetal pulmonary vein flow has been described as a systolic and early diastolic forward flow followed by a late-diastole forward flow component [7]. Interestingly, a recent case series suggested an enhanced first trimester screening for CHD, when adding the presence of pulmonary vein (Awave) flow reversal [8]. However, only inconsistent data exist about the acquisition of pulmonary vein flow in early pregnancy $[6,9]$.

The present study aims to determine: (a) the feasibility of fetal pulmonary vein flow detection by Doppler ultrasound during the first trimester of gestation, and (b) the potential association between pulmonary vein A-wave reversal and presence of $\mathrm{CHD}$.

\section{Materials and Methods}

A total of 211 consecutive pregnant women, between 12 and 15 weeks of gestation, were prospectively enrolled between 2012 and 2013 at "Centro de Estudios Ultrasonograficos Perinatales." Valencia, Venezuela. This specialized fetal ultrasound unit functions as a moderate to high-risk pregnancy referral center. Patients underwent routine obstetric ultrasound along with basic fetal echocardiography. The obstetric ultrasound included fetal biometry, nuchal translucency, and central nervous system examination. Meanwhile, the heart screening comprised fetal heart rate monitoring and fetal echocardiography: four-chamber view, outflow-tract view, and pulse wave Doppler of (a) the right-upper pulmonary vein, (b) ductus venosus, and (c) tricuspid and mitral inflows. The ultrasound was performed by either the transabdominal and/or transvaginal approach at the physician's discretion; the latter approach was used when in the presence of maternal obesity, early gestation age, and/or poor transabdominal ultrasound image quality. The use of Doppler alone versus Doppler plus enhance flow technique was determined by equipment availability. Four physicians with prenatal ultrasound experience $(\mathrm{GG}, \mathrm{DL}, \mathrm{BA}$, and S-OA) performed the obstetric ultrasounds. The equipment included MyLab Class eHD ESAOTE, Samsung Medison XG, Accuvix V20 Medison, and Voluson 730 expert GE.

All the cases were re-evaluated by specialized fetal echocardiography during late second or third trimesters of gestation. Four cases with CHD had perinatal autopsy (S-OA) to better describe the cardiac anomaly. The local ethics committee approved the study, all patients consented to participate in the study, and the ALARA principle was always honored.

\section{Acquisition of Fetal Pulmonary Venous Flow Velocity Waveforms by Doppler}

Via either transabdominal or transvaginal ultrasound, based on gestational age, body habitus and image quality, the four-chamber view was obtained. The right upper pulmonary vein was localized at the left atrium entrance, at the upper segment of the left atrium above the interatrial septum. The spine, sternum, descendant aorta, and gastric camera were used as important landmarks. An insonation angle of less than $15^{\circ}$ with a sample volume between 0.1 and $0.3 \mathrm{~cm}$ was used to obtain the spectral wave of the pulmonary vein. The low flow filter and very low pulmonary reversal flow (PRF) were adjusted accordingly. When using Doppler without enhanced-flow (158 fetuses), the sample volume was placed directly in the anatomic area of the pulmonary vein entrance to the left atrium. While, when using Doppler with enhanced flow technique (70 fetuses), the color box was adjusted to the cardiac area with gaining graduation, low PRF, and low flow filter.

The pulmonary vein was identified by: (1) obtaining similar waveforms to the vascular flow of the ductus venosus, and (2) confirming flow direction toward the left atrium. After few uniform waves were obtained, the use of B-REF in the spectral mode increased and optimized the reading and interpretation of the flow velocity waveforms (FVWs).

The main limitations for the pulmonary vein detection were excess of fetal movements and the small size of the cardiac structures. Similarly, in a few occasions, the pulmonary vein signals were distorted by an overlapped pulmonary artery signal.

\section{Statistics}

The detection rates of pulmonary venous FVWs between Doppler with and without enhanced flow technique were compared using Chi square with Yates correction. The association between A-wave reversal and the presence of cardiac anomaly was reported using Chi square.

\section{Results}

All 211 pregnant women enrolled in the study were included in the final analysis. Only one patient was lost to follow-up and analyzed as intention to treat. The mean age was 30 years $(\mathrm{SD} \pm 5.25)$. Most pregnant women $(85 \%)$ were between 13 and 14 weeks of gestation. The most common maternal comorbidities were: hypothyroidism (10 cases), gemelar pregnancy (8), obesity (7), arterial hypertension (7), hyperinsulinism (6), and diabetes mellitus (1) (Table 1). 
Table 1 Demographics divided by ultrasound method used for pulmonary vein flow acquisition

\begin{tabular}{|c|c|c|c|c|}
\hline & Doppler US ${ }^{\mathrm{a}}$ & Doppler US ${ }^{\mathrm{a}}$ plus $\mathrm{X}$-flow & Overall & p-value \\
\hline Maternal age (y) (Mean \pm SD) & $34 \pm 2.87$ & $30 \pm 5.14$ & $30 \pm 5.25$ & $<0.05$ \\
\hline Gestational age (w) (Mean \pm SD) & $13.46 \pm 0.65$ & $13.41 \pm 0.69$ & $13.46 \pm 0.74$ & 0.59 \\
\hline \multicolumn{5}{|l|}{ Race } \\
\hline Hispanic & $141(89.2 \%)$ & $58(82.8 \%)$ & $184(87.2 \%)$ & 0.27 \\
\hline White & $14(8.9 \%)$ & $12(17.1 \%)$ & $24(11.4 \%)$ & 0.13 \\
\hline African American & $1(0.6 \%)$ & 0 & $1(0.5 \%)$ & - \\
\hline Other & $2(1.3 \%)$ & 0 & $2(0.9 \%)$ & - \\
\hline \multicolumn{5}{|l|}{ Comorbidities } \\
\hline Arterial hypertension & 5 & 2 & 7 & - \\
\hline Type 2 diabetes mellitus & 1 & 0 & 1 & - \\
\hline Gestational diabetes & 1 & 0 & 1 & - \\
\hline Hyperinsulinism & 7 & 2 & 8 & - \\
\hline Hypothyroidism & 9 & 1 & 10 & - \\
\hline Obesity & 3 & 2 & 5 & - \\
\hline Anti-phospholipid syndrome & 2 & 1 & 3 & - \\
\hline Hyperprolactinemia & 1 & 0 & 1 & - \\
\hline Achondroplasia & 1 & 0 & 1 & - \\
\hline Gemelar fetuses & 6 & 4 & 8 & - \\
\hline ABO incompatibility & 3 & 1 & 4 & - \\
\hline $\mathrm{Rh}$ incompatibility & 2 & 1 & 3 & - \\
\hline Epilepsy & 0 & 1 & 1 & - \\
\hline
\end{tabular}

a Ultrasound

Fetal screening, across patients enrolled, demonstrated a rate of enlarged nuchal translucency of $1.9 \%$. A limited four-chamber view was obtained in 186 cases, while basic outflow tract view was only registered in 59 cases. Doppler assessment revealed a ductus venosus reversal in $1.4 \%$ of cases, and abnormal tricuspid inflow in $0.9 \%$ fetuses, but none with anomalous mitral inflow. The studied cohort had an incidence of fetal cardiac anomalies of $2.8 \%$, with 5 cases described as CHD, and 1 case with functional cardiomyopathy.

\section{Acquisition of the Pulmonary Venous Flow Velocity Waveform}

Out of the 211 enrolled patients, 158 cases underwent pulmonary vein interrogation by Doppler alone, while 70 had Doppler with enhanced-flow technique. The acquisition of fetal pulmonary vein, between 12 and 15 weeks of gestation, was successfully accomplished in $87.7 \%$ of cases. When reporting acquisition by technique, Doppler with enhanced-flow technique registered $90 \%$ of pulmonary veins interrogated; while Doppler alone detected $86.7 \%$ (Table 2). Understanding the limitation in patient allocation into Doppler with or without enhanced flow due to equipment availability, this study suggests no difference between the two methods for the acquisition of fetal pulmonary venous FVW in early pregnancy ( $\mathrm{p}$-value $=0.63$ ).

The pulmonary vein wave was properly described by Doppler alone in $85.2 \%$ of cases (75/88 fetuses) at 13 weeks of gestation and $86.9 \%$ (40/46 fetuses) of cases at 14 weeks. When adding enhanced flow, pulmonary vein flow was described in $96.8 \%$ of cases (31/32 fetuses) at 13 weeks, and $86.2 \%(25 / 29)$ of the cases at 14 weeks.

\section{End-Diastolic (A-wave) Pulmonary Venous Flow Reversal and Congenital Heart Disease}

In this study, the pulmonary venous waveforms, during early pregnancy, demonstrated a forward flow during systole, and early diastole, with a nadir during atrial systole. Upon characterization of pulmonary venous (A-wave) waveform, forward flow was reported in $91.4 \%$ of cases, whereas absence of flow and flow reversal were described in $4.8 \%$ and $3.7 \%$ of the cases, respectively (Fig. 1).

Five out of seven fetuses with end-diastolic (A-wave) pulmonary venous flow reversal were associated with confirmed cardiac anomaly by second or third trimester echocardiography (Table 3). Pulmonary venous A-wave flow reversal was associated with cardiac anomalies in $86.8 \%$ of cases $(\mathrm{p}=0.001)$. The sixth case was lost during follow-up, 
Table 2 Rate of detection of pulmonary vein flow between 12 and 15 weeks of gestation by Doppler technique

\begin{tabular}{|c|c|c|c|c|}
\hline & Attempt & Found & Not found & $\begin{array}{l}\text { Rate of } \\
\text { detection }(\%)\end{array}$ \\
\hline Doppler US ${ }^{\mathrm{a}}$ & 158 & 137 & 21 & 86.71 \\
\hline Doppler US ${ }^{\mathrm{a}}$ plus X-flow & 70 & 63 & 7 & 90.00 \\
\hline
\end{tabular}

${ }^{\mathrm{a}}$ Ultrasound

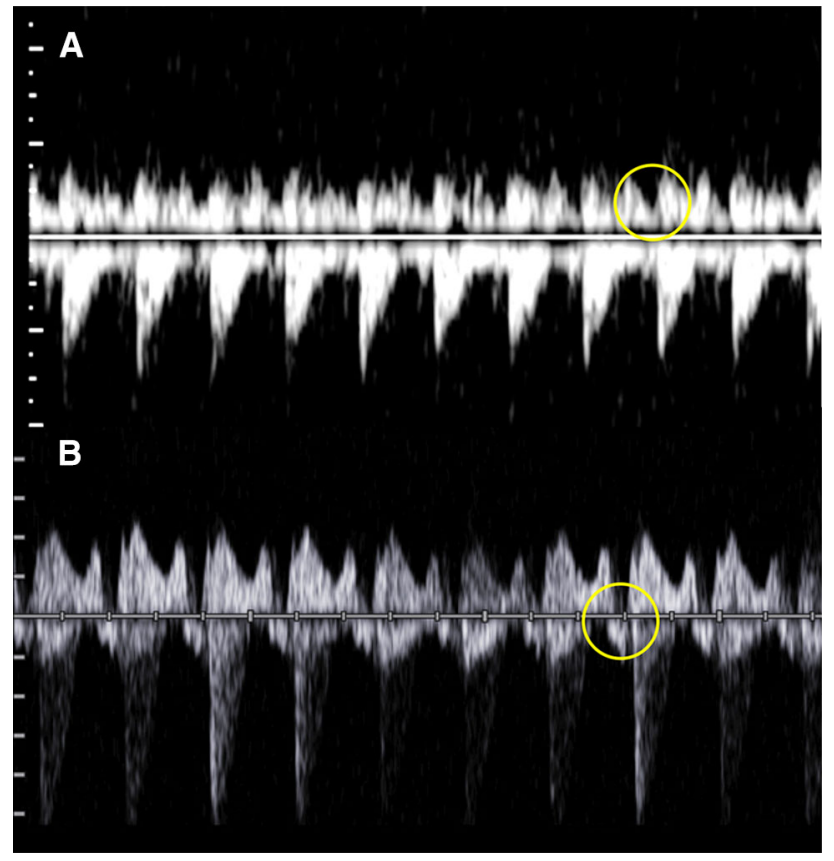

Fig. 1 Flow velocity waveforms of fetal pulmonary vein during early pregnancy by Doppler (a) Circle encases forward flow during pulmonary venous (A-wave) flow, noticed that flow velocity remains above baseline while the signal below baseline represents the overlapped pulmonary artery flow (b) Circle shows pulmonary venous (A-wave) flow reversal, notice that the flow velocity dips below baseline

but early echocardiography was suggestive of aortic coarctation, while the seventh case was a normal fetus. Overall, pulmonary venous (A-wave) flow reversal was present in only $0.55 \%$ of normal fetuses. Meanwhile, only one case with normal pulmonary venous FVW presented with major cardiac anomaly. The defect was described as a tricuspid atresia with a ventricular septal defect (VSD), and a large atrial shunt.

\section{Discussion}

This pilot study proposes that the acquisition of fetal pulmonary venous flow is feasible during early pregnancy, by either pulsed Doppler or Doppler with enhanced-flow (Xflow) technique. Furthermore, the results suggest no difference in the rate of pulmonary vein detection between the two techniques. However, this statement must be carefully interpreted since the lack of consistent patient allocation into the two techniques, due to equipment availability, might have biased the results.

\section{Detection of Fetal Pulmonary Venous Flow Waveforms during Early Pregnancy}

Scant data exist about the description of pulmonary venous FVW before 17 weeks of gestation [10]. Our study reported a high acquisition rate $(87.7 \%)$ of pulmonary venous flow in early pregnancy. Previous studies have interrogated the fetal pulmonary veins, but with lower rates of success. First, Dong et al. [9] reported a detection rate of 12 out of $29(41 \%)$ cases with gestation ages between 12 and 22 weeks. However, there was no transvaginal ultrasound use, and a positive detection was recorded only when all four pulmonary veins were characterized. Therefore, these two factors could have accounted for the difference in the rate of pulmonary vein detection.

Subsequently, Moon-Grady et al. [6] reported fetal pulmonary vein detection rates of $0 \%$ at 12 weeks, $50 \%$ at 13 weeks, $85 \%$ at 14 weeks, $56 \%$ at 15 weeks, and $50 \%$ at 16 weeks. The rate of detection at 14 weeks was similar to our study. Yet, detection declined at 15 and 16 weeks of gestation suggesting against gestational age as the main limiting factor. Instead, technical deficiencies could be summoned since reports of inability to localize the pulmonary vein existed, along with difficulties to place sample volume for Doppler interrogation. Also, the small sample at gestational ages other than 14 weeks could have altered the results.

The pulmonary venous waveform during early pregnancy was described as a pulsatile flow with systolic and diastolic forward flow, even during atrial systole. This mirrors the flow patterns described in late pregnancy by previous studies [7, 10,11]. A pulmonary vein A-wave reversal was only described in $0.55 \%$ of normal fetuses. Similar low rates have been described in the literature. While, Lenz et al. $[10,11]$ reported no reversal across normal fetuses, Dong et al. [9] reported a rate of A-wave reversal of $3.3 \%$ among normal fetuses.

\section{End-Diastolic (A-wave) Pulmonary Venous Flow Reversal and Congenital Heart Disease}

The studied cohort had a higher than normal $2.8 \%$ incidence of fetal cardiac anomalies (6 out of 211). Our center 
Table 3 First trimester screening markers of cases with confirmed congenital heart anomaly

\begin{tabular}{|c|c|c|c|c|c|c|c|}
\hline & AV canal & $\begin{array}{l}\text { Aortic } \\
\text { coarctation }\end{array}$ & $\begin{array}{l}\text { Aortic arch } \\
\text { interruption }\end{array}$ & VSD & $\begin{array}{l}\text { LV } \\
\text { hypoplasia }\end{array}$ & $\begin{array}{l}\text { Dilated } \\
\text { cardiomyopathy }\end{array}$ & $\begin{array}{l}\text { Tricuspid } \\
\text { atresia }\end{array}$ \\
\hline Gestational age (weeks) & 13 & 14 & 12 & 13 & 13 & 13 & 12 \\
\hline \multicolumn{8}{|l|}{ Structural } \\
\hline $\begin{array}{l}\text { Nuchal translucency } \\
(\mathrm{mm})\end{array}$ & 6 & Normal & Normal & Normal & 6 & 3.2 & Normal \\
\hline \multicolumn{8}{|l|}{ Doppler US } \\
\hline Ductus venosus & Reversal & Normal & Reversal & Normal & Reversal & Normal & Normal \\
\hline Tricuspid inflow & Normal & Normal & Normal & Normal & Regurgitation & Normal & Normal \\
\hline Mitral inflow & Normal & Normal & Normal & Normal & NA & NA & Normal \\
\hline Pulmonary vein & Reversal & Reversal & Reversal & Reversal & Reversal & Reversal & Normal \\
\hline
\end{tabular}

$A V$ atrioventricular, $C M$ cardiomyopathy, $L V$ left ventricular, $U S$ ultrasound, $V S D$ ventricular septal defect

serves as a high-risk pregnancy referral center with impact over surrounding states; we propose the volume of highrisk pregnancies referred to our center as the main factor accounting for the mentioned extraordinary incidence.

In this pilot study, the presence of pulmonary venous (A-wave) flow reversal seemed to be associated with the existence of cardiac anomaly $(p=0.001)$. Similarly, Lenz et al. [11] described A-wave pattern in pulmonary vein in presence of cardiac anomalies. A pulmonary venous enddiastolic flow reversal was described mostly with atrial obstructing anomalies, but also those cases with severe left ventricle outflow obstructions. Nevertheless, miscellaneous anomalies, such as VSD and atrial septal defect, had normal pulmonary vein flow contrasting with our findings. Yet, the study by Lenz et al. [11] was conducted in pregnant women during mid-late pregnancy, therefore an improved compensatory capacity with increasing gestational age could be summoned as an explanation to the normalization of the pulmonary flow.

Only one case had normal pulmonary vein in the presence of a cardiac anomaly. The heart defect was described as a right ventricle hypoplasia with tricuspid atresia, VSD and patent foramen ovale. This finding is supported by the study by Lenz et al. [11] where all right side anomalies presented with normal end-diastolic pulmonary venous flow. We propose that alteration in pulmonary venous flow might mainly reflect left heart anomalies. Finally, an anterograde or at baseline pulmonary vein A-waves were associated with normal second or third trimester echocardiography.

\section{Study Limitation}

The absence of randomization and blinding may have introduced bias. The highly experienced ultrasonographers conducting the study, the use of different ultrasounds machines, and the lack of standardization in the use of transvaginal ultrasound could compromise the reproducibility of the study. The use of Doppler alone versus Doppler with the addition of enhanced-flow was not uniform across due to equipment availability. Moreover, the small sample of fetuses with cardiac anomalies could have altered the association between the pulmonary veins and cardiac anomalies. Nevertheless, the aim of the study was not to provide data to change clinical practice but to offer a basis for future research.

\section{Conclusion}

The detection of the pulmonary venous flow by Doppler during the first trimester seems feasible. Moreover, the presence of pulmonary venous (A-wave) flow reversal might represent an early marker of major cardiac anomalies. Further investigation is needed to confirm these findings.

Conflict of interest None.

\section{References}

1. ISUOG Practice Guidelines (updated). Sonographic screening examination of the fetal heart. Ultrasound Obstet Gynecol. 2013;4:348-59.

2. Rasiah SV, Publicover M, Ewer AK, et al. A systematic review of the accuracy of first-trimester ultrasound examination for detecting major congenital heart disease. Ultrasound Obstet Gynecol. 2006;28:110-6.

3. Simpson J. Impact of fetal echocardiography. Ann Pediatr Cardiol. 2009;2(1):41-50.

4. Pinto NM, Keenan HT, Minich LL, et al. Barriers to prenatal detection of congenital heart disease: a population-based study. Ultrasound Obstet Gynecol. 2012;40:418-25. 
5. Prakash A, Powell AJ, Geva T. Multimodality noninvasive imaging for assessment of congenital heart disease. Circ Cardiovasc Imaging. 2010;3(1):112-5.

6. Moon-Grady A, Shahanavaz S, Brook M, et al. Can a complete fetal echocardiogram be performed at 12-16 weeks of gestation? J Am Soc Echocardiogr. 2012;25(12):1342-52.

7. Laudy JA, Huisman TWA, de Ridder MA. Normal fetal pulmonary venous blood flow velocity. Ultrasound Obstet Gynecol. 1995;6:277-81.

8. Schenone AL, Giugni G, Schenone MH. Case series: fetal pulmonary vein a-wave reversal: an early marker of left-sided cardiacanomalies? Am J Perinatol Rep.
9. Dong FQ, Zhang YH, Li ZA, et al. Evaluation of normal fetal pulmonary veins from the early second trimester by enhancedflow (e-flow) echocardiography. Ultrasound Obstet Gynecol. 2011;38(6):652-7.

10. Lenz F, Chaoui R. Reference ranges for Doppler-assessed pulmonary venous blood flow velocities and pulsatility indices in normal human fetuses. Prenat Diagn. 2002;22:786-91.

11. Lenz F, Chaoui R. Changes in pulmonary venous Doppler parameters in fetal cardiac defects. Ultrasound Obstet Gynecol. 2006;28:63-70. 